\title{
Percepción y valoración de variedades geográficas del español de Chile entre hispanohablantes santiaguinos*
}

\author{
Darío Rojas ** \\ Universidad de Chile
}

\begin{abstract}
Resumen
En el presente artículo describimos la percepción que tienen los hispanohablantes santiaguinos de la variación geográfica del español de Chile en términos de tres dimensiones valorativas: la similitud que observan respecto del habla propia en el habla de distintos lugares, la calidad que atribuyen a las variedades percibidas y el agrado que sienten por ellas. Estas percepciones revelan las actitudes de los sujetos hacia las variedades geográficas que identifican en el español chileno. Concluimos que, desde la perspectiva intranacional, los santiaguinos poseen un alto grado de autoestima lingüística, pues su actitud hacia el habla de la capital y la zona central, en general, es más positiva que la que muestran hacia las zonas norte y sur. Sin embargo, en el dominio afectivo (agrado) y entre los segmentos medios y bajos de la escala socioeconómica, el habla de la zona sur
\end{abstract}

* Esta investigación se enmarca en el proyecto NFR-193742, Linguistic Identity and Attitudes in Spanish-speaking Latin America - LIAS (Identidad y actitudes lingüísticas en Hispanoamérica - LIAS), financiado por el Research Council of Norway (Consejo Noruego de Investigación).

** Para correspondencia, dirigirse a: Darío Rojas (dariorojasgall@hotmail.com), Departamento de Lingüística, Facultad de Filosofía y Humanidades, Universidad de Chile, Ignacio Carrera Pinto 1025, Nuñoa, Santiago, Chile. 
de Chile incrementa su valoración positiva, aunque sin superar la de la zona central del país.

Palabras clave: español de Chile, actitudes lingüísticas, variación geolingüística, dialectología, lingüística popular.

\title{
Perception AND VAluation of GeOgRaPhicAl VARIETIES OF Chilean Spanish amongst Spanish-SPeaking subjects from SANTiago de Chile
}

\begin{abstract}
In this paper we describe the perception which Spanish-speaking subjects from Santiago de Chile have of the geographical variation of Chilean Spanish in terms of three dimensions: the similarity observed in the speech of various regions in relation to their own speech, the quality attached to regional varieties, and the pleasantness perceived in them. These perceptions reveal the attitudes that the subjects have towards the regional varieties of Chilean Spanish identified by themselves. We conclude that people from Santiago de Chile have a high degree of linguistic self-esteem as they show a more favourable attitude towards the variety of Spanish spoken in the capital city and in the central region of Chile, in general, than towards the varieties spoken in the northern and southern regions. Nevertheless, in the affective dimension (pleasantness) and specially amongst people from the middle and lower classes, the southern variety increases its positive valuation, although never surpassing the positive valuation of the central region of this country.
\end{abstract}

Key words: Chilean Spanish, linguistic attitudes, geolinguistic variation, dialectology, folk linguistics.

Recibido: 26/12/11 Aceptado: 03/05/12

\section{INTRODUCCIÓN}

El estudio de las actitudes lingüísticas ocupa un lugar central en la comprensión de la relación entre lenguaje y sociedad. A pesar de su importancia, en el ámbito hispánico las investigaciones sobre el tema aún 
son escasas y de alcance parcial (Blas Arroyo 1999). Aunque no se puede negar que este asunto ha despertado el interés de los estudiosos, hasta la fecha no se ha llevado a cabo ningún proyecto de investigación de grandes dimensiones que recoja materiales sobre actitudes lingüísticas hacia el español y sus variedades, en distintos lugares del mundo hispanohablante, con una metodología común que permita comparar entre sí los resultados de diversas zonas. En Chile, las investigaciones relativas al tema se han enfocado en algún rasgo lingüístico en particular o han trabajado con muestras pequeñas (Figueroa 2007; Tassara 1992; Tassara 1993-1994; Valdivieso 1981; Valdivieso 1983). En consecuencia, existe un gran vacío de conocimientos sobre las valoraciones subjetivas respecto del empleo del español tanto en un nivel global como en un nivel local. Los resultados finales del proyecto Linguistic Identity and Attitudes in Spanish-speaking Latin America (en adelante, LIAS), de la Universidad de Bergen (Noruega), contribuirán de manera importante a llenar este vacío. En el marco del proyecto $L I A S$, el primero de alcance global en su tipo, durante los años 2009 y 2010 se han registrado y estudiado las actitudes lingüísticas de hispanohablantes provenientes de 21 ciudades del mundo hispánico, la mayoría correspondientes a capitales. En total, se han aplicado 8400 encuestas (400 por ciudad) a muestras de individuos estratificados de acuerdo con edad, sexo y grupo socioeconómico. En Chile, se escogió entrevistar a sujetos de Santiago de Chile, tanto por concentrar una parte considerable de la población del país como por su carácter de centro irradiador de modelos de pensamiento y conducta (para lo lingüístico, $c f$. Oroz 1966: 49 y Prieto 1995-1996: 380-382).

En el presente artículo mostramos resultados parciales de esta investigación. Examinamos la percepción que tienen los santiaguinos de las variedades del español de Chile en términos de tres dimensiones valorativas: la similitud que observan respecto del habla propia, la calidad que atribuyen a las variedades percibidas y el agrado que sienten por ellas. A través de estas percepciones, nos proponemos determinar las actitudes que muestran los hispanohablantes santiaguinos hacia las formas de hablar español dentro del territorio chileno.

Como es obvio, la descripción de las percepciones señaladas presupone que los encuestados revelen cuáles son las variedades geográficas internas que distinguen en el español chileno, es decir, la zonificación lingüística folk subyacente a estas percepciones. Sin embargo, ninguna de las preguntas del cuestionario estaba dirigida expresamente a obtener dicha información (no había una pregunta del tipo "¿Qué zonas o dialectos percibe usted en el español de Chile?"), por lo cual en la presente investigación no nos haremos cargo de este asunto, que creemos debe ser abordado en un trabajo 
independiente y con un método ad hoc (como el que ofrece la dialectología perceptual; Preston 1999; Long y Preston 2002).

\section{MARCO CONCEPTUAL}

Entendemos por actitud lingüistica la valoración positiva o negativa que un individuo o grupo muestra hacia un rasgo lingüístico, una variedad lingüística completa o los usuarios de dicho rasgo o variedad. En las actitudes lingüísticas se pueden distinguir tres elementos estructurales: un componente cognitivo, es decir, creencias (ciertas o no ciertas) sobre el lenguaje y sobre el mundo en general; uno afectivo, vale decir, las actitudes involucran sentimientos y emociones que tienen por objeto el lenguaje; por último, un elemento conductual (o conativo), esto es, una predisposición a actuar de cierto modo respecto del objeto de la actitud (Garrett 2010: 19-20; Trudgill y Hernández 2007: s. v. actitudes lingüisticas).

Las creencias que subyacen a las actitudes lingüísticas deben ser entendidas, como han apuntado varios autores (Irvine y Gal 2000; Milroy y Milroy 1999; Schieffelin, Woolard y Kroskrity 1998), y en la medida en que se encuentren articuladas en sistemas de pensamiento, como parte de ideologías lingüisticas ${ }^{1}$ o culturas lingüisticas ${ }^{2}$ que se enmarcan, a su vez, en contextos socioculturales específicos (Coupland y Jaworski 2004: 37). En la cultura lingüística hispánica (caracterizada por Del Valle y GabrielStheeman 2002 como una ideología de tipo monoglósico), ocupan un lugar central los conceptos de unidad y de corrección idiomática (Coseriu 1990; Coseriu 1993). En particular, puede presumirse que la mayor o menor corrección que los sujetos inmersos en esta cultura lingüística perciben en ciertos rasgos idiomáticos o en variedades del español los predispone a valorar dichos rasgos o variedades de manera positiva (a mayor corrección) o negativa (a menor corrección).

1 "Representations, whether explicit or implicit, that construe the intersection of language and human beings in a social world" (Woolard 1998: 3).

2 “... a relatively abstract and supposedly universal set of beliefs about general concepts such as language, speech, speech community, literacy, etc." (Del Valle y Gabriel-Stheeman 2002: 10). 
Sin embargo, a pesar de su carácter predominante (junto con la dimensión del agrado, según Preston 2002), la corrección es solo una de las dimensiones en que pueden ser valorado el lenguaje y los hablantes. En el marco de los estudios de actitudes lingüísticas que utilizan el método indirecto (en especial la técnica de pares falsos; $c f$. Garrett 2010: 39-46), se ha logrado identificar varias dimensiones valorativas recurrentes y se ha propuesto agruparlas en macrodimensiones. Zahn y Hopper (1985) consideran que las tres principales macrodimensiones de valoración del lenguaje son la superioridad (educación, riqueza, etc.), el atractivo social (amigabilidad, honestidad, etc.) y el dinamismo (energía, entusiasmo, etc.). Edwards (2011: 62) indica, basado en una amplia cantidad de estudios sobre este asunto, que las principales macrodimensiones serían la competencia (inteligencia, etc.), integridad personal (confiabilidad, etc.) y el atractivo social (amigabilidad, sentido del humor, etc.); no obstante, investigaciones recientes sugieren que podrían establecerse simplemente dos grandes categorías: estatus social, más o menos equivalente a la competencia, y solidaridad, que aunaría integridad y atractivo social. Bayard y otros (2001), por su parte, agrupan las valoraciones identificadas en su estudio de acuerdo con las siguientes esferas: poder (control, autoridad, asertividad, etc.), competencia (inteligencia, educación, confiabilidad, etc.), solidaridad (amigabilidad, calidez, alegría, etc.) y estatus (ingreso, clase social, etc.). En un estudio realizado desde la aproximación teórico-metodológica de la lingüística folk, Garrett y otros (2005) clasifican las palabras clave de carácter evaluativo elicitadas en sus informantes de acuerdo con cuatro categorías: rasgos lingüísticos (descripciones no técnicas de diversas características fónicas, gramaticales, léxicas o discursivas), afecto ("agradable", "divertido", etc.), estatus y normas sociales ("inteligente", "correcto", etc.) y asociaciones culturales (por ejemplo, "McDonalds" para el inglés de los Estados Unidos). En el ámbito hispánico, Blas Arroyo (1995) considera como principales las siguientes macrodimensiones: atractivo personal (bondad, humildad, lealtad, etc.), atractivo social (modernidad, alegría, etc.) y estatus socioeconómico (corrección, riqueza, inteligencia, cultura, etc.). González Martínez (2008) añade, a las identificadas por Blas Arroyo, la macrodimensión de la cercanía del sistema (claridad y familiaridad). Coseriu (1993), en su minucioso análisis del lugar de la corrección entre los juicios de lo hablado, distingue entre juicios lingüísticos (por ejemplo, "correcto") y juicios no lingüísticos (por ejemplo, "bello", "inteligente", "alegre", etc.); la mayor parte de las dimensiones valorativas identificadas en estos estudios sobre actitudes son de tipo no lingüístico, es decir, "valoran otros aspectos del hablar en cuanto acción, en cuanto a manifestación del ser, del hablante, de la cultura del hablante, etc." (Coseriu 1993: 63). Esto no puede sorprendernos, pues es sabido que las actitudes 
hacia variedades o rasgos con mucha frecuencia reflejan actitudes hacia sus usuarios, más que hacia una pronunciación, uso gramatical o término léxico en particular (Milroy 2001: 532; Edwards 2011: 60-61).

En nuestro estudio hemos seleccionado tres dimensiones valorativas para abordar el problema de la percepción de la variación geográfica del español de Chile: la similitud (o diferencia) percibida en un habla respecto de la propia, la calidad percibida en una variedad diatópica (lo cual fue planteado por los encuestadores en términos de mejor/peor) y el agrado (o desagrado) suscitado por cada una de estas modalidades del hablar. Estas tres dimensiones representan bien las facetas cognitiva (similitud y calidad) y afectiva (agrado) de las actitudes lingüísticas.

En cuanto al carácter positivo o negativo de las actitudes que revelan estas valoraciones, establecemos la siguiente correlación:

Cuadro 1. Valores positivos y negativos de cada dimensión utilizada en el estudio

\begin{tabular}{|c|c|c|}
\hline Dimensión & Valoración positiva & Valoración negativa \\
\hline similitud & similar & diferente \\
calidad & mejor & peor \\
agrado & agradable & desagradable \\
\hline
\end{tabular}

La precisión de estas correlaciones se encuentra suficientemente probada por numerosos estudios empíricos (como los reseñados en Garrett 2010), en especial la correlación relativa al agrado. Sin embargo, conviene hacer un comentario más detallado en relación con la calidad y, particularmente, en el caso de la similitud.

Respecto de la calidad, podría objetarse que el hablar "mejor" no es siempre valorado positivamente, pues no son pocos los estudios que revelan el prestigio encubierto conferido por ciertas subcomunidades a formas poco prestigiosas, consideradas representativas del hablar "mal". No obstante, sí es cierto que al menos en el nivel de la esfera pública, al nivel del prestigio manifiesto, existe una fuerte asociación entre el hablar "bien" y la valoración positiva: por ejemplo, hablar "correctamente" se correlaciona fuertemente con actitudes positivas. El método aplicado en nuestra investigación es revelador de actitudes de nivel consciente (Kristiansen 2010: 60-61), nivel en el que predomina el peso del prestigio manifiesto, con lo cual podemos presumir que la correlación que hemos establecido es cierta para nuestro estudio. 
En cuanto a la similitud, podría argumentarse que se trata de una valoración neutra, es decir, ni positiva ni negativa. Sin embargo, es bien sabido que en la cultura lingüística hispánica tradicionalmente ha tenido una gran importancia la unidad del idioma (Gallardo 2009-2010; MenéndezPidal 1945), por lo que la variedad y la diferencia, que en principio atentarían contra la unidad, tienden a ser miradas de manera negativa o, al menos, percibidas con preocupación (para los antecedentes románicos medievales de esta visión, $c f$. Zimmermann 2008). Irvine y Gal (2000) señalan que uno de los procesos semióticos recurrentes a través de los cuales se gestan ideologías lingüísticas es la elisión (erasure), es decir, la simplificación de la variación mediante la invisibilización de algunos actores o fenómenos lingüísticos diferentes a los hegemónicos. La frecuencia con que la diferencia es objeto de esta operación semiótica (y su ubicuidad en la cultura lingüística hispánica), entonces, puede considerarse reveladora de que, al menos en la esfera del discurso oficial o público hispánico, es considerada como algo negativo ${ }^{3}$. En segundo lugar, teniendo en cuenta el importante papel que desempeña el lenguaje en la conformación y el mantenimiento de identidades grupales (Edwards 2009), si un sujeto considera un objeto lingüístico (un rasgo o variedad) como similar al que es propio de su grupo puede presumirse que se sentirá identificado con él $\mathrm{y}$, consecuentemente, tendrá una actitud positiva hacia el mismo. A la inversa, si percibe el mismo objeto como diferente, no tendrá una base para la identificación y desarrollará una disposición negativa hacia él. Esto se condice con la tendencia sociosicológica denominada favoritismo intragrupal (Myers 2010: 326-328; Edwards 2009: 26-27). Igualmente, uno de los principales hallazgos de los estudios sobre acomodación lingüística es que "we like others who are like ourselves" (Edwards 2011: 64). En conclusión, por las dos razones explicadas, creemos justificada la correlación entre, por un lado, percepción de similitud y actitud positiva y, por otro, diferencia y actitud negativa.

3 Piénsese, por ejemplo, en la tradición de los diccionarios de regionalismos hispanoamericanos, que consideraban la mayor parte de los usos idiomáticos locales como "vicios del lenguaje" (Haensch 2000). Aún hoy este punto de vista es muy común en las opiniones de los legos. 


\section{METODOLOGÍA}

\subsection{Sujetos DE LA MUESTRA}

La muestra de sujetos encuestados se compone de 400 individuos, agrupados de acuerdo con estrato socioeconómico (bajo, medio y alto ${ }^{4}$ ), grupo etario (20 a 34, 35 a 54 y 55 o más años) y sexo (hombres y mujeres). La representación de cada uno de los estrato y grupos es proporcional a la que tienen en el universo de la población nacional ${ }^{5}$.

Tabla 1. Distribución de los sujetos de la muestra según estrato socioeconómico, grupo etario y sexo

\begin{tabular}{|c|c|c|c|}
\hline Estrato & Grupo etario & Sexo & $\mathrm{n}$ \\
\hline \multirow{6}{*}{ Bajo } & \multirow{2}{*}{$20-34$} & Hombres & 31 \\
\hline & & Mujeres & 34 \\
\hline & \multirow{2}{*}{$35-54$} & Hombres & 35 \\
\hline & & Mujeres & 38 \\
\hline & \multirow{2}{*}{55 o más } & Hombres & 20 \\
\hline & & Mujeres & 22 \\
\hline \multirow{6}{*}{ Medio } & \multirow{2}{*}{$20-34$} & Hombres & 31 \\
\hline & & Mujeres & 34 \\
\hline & \multirow{2}{*}{$35-54$} & Hombres & 35 \\
\hline & & Mujeres & 38 \\
\hline & \multirow{2}{*}{55 o más } & Hombres & 20 \\
\hline & & Mujeres & 22 \\
\hline
\end{tabular}

4 Para la determinación de los estratos socioeconómicos empleamos los datos de ICCOM (Instituto Consultor en Comercialización y Mercado Limitada), en su versión del año 2007 (tablas disponibles en www.iccom.cl). Las equivalencias y proporciones son las siguientes: estrato alto $=\mathrm{ABC} 1$; estrato medio $=\mathrm{C} 2+\mathrm{C} 3$; estrato bajo $=\mathrm{D}+\mathrm{E}$.

5 Según el censo nacional del año 2002 (www.ine.cl). Las proporciones son las siguientes: estrato alto $=11 \%$, estrato medio $44,5 \%$, estrato bajo $44,5 \% ; 20-34$ años $=36 \%, 35-54$ años $=40,5 \%, 55$ años o más $=23,5 \%$; hombres $=48 \%$, mujeres $=52 \%$. 


\begin{tabular}{|c|c|c|c|}
\hline \multirow{4}{*}{ Alto } & \multirow{2}{*}{$20-34$} & Hombres & 7 \\
\cline { 2 - 4 } & & Mujeres & 7 \\
\cline { 2 - 4 } & \multirow{2}{*}{$35-54$} & Hombres & 8 \\
\cline { 2 - 4 } & \multirow{2}{*}{55 o más } & Mujeres & 8 \\
\cline { 2 - 4 } & & Hombres & 5 \\
\hline & & & 5 \\
\hline
\end{tabular}

Todos los sujetos encuestados viven en el núcleo urbano del Gran Santiago ${ }^{6}$ desde hace 20 años o más (la mayor parte lo han hecho toda su vida). Aunque no asignamos una cuota de acuerdo con la representatividad demográfica de cada comuna, la mayoría proviene de un grupo de comunas que resultan prototípicas de cada estrato social. Así, por ejemplo, la mayor parte de los individuos de estrato alto proceden de Vitacura, Las Condes y Lo Barnechea, mientras que la mayor parte de los de estrato bajo proceden de La Pintana, Cerro Navia, San Ramón y Quilicura?

\subsection{INSTRUMENTO DE RECOLECCIÓN DE DATOS Y APLICACIÓN}

Para observar las actitudes lingüísticas de los sujetos se utilizó el método directo (Garrett 2010: 159-178). El instrumento utilizado para recoger los datos consiste en un cuestionario de 40 preguntas. Algunas de ellas corresponden a los datos sociodemográficos de los encuestados: las preguntas que miden directamente las actitudes de los hablantes son 26. Estas elicitan opiniones sobre el español del propio país (incluida su variación interna), sobre el español de otros países (también en comparación con el propio) y sobre el español general. El cuestionario incluye tanto preguntas de respuesta cerrada como preguntas de respuesta abierta, ambas con espacio para explicaciones por parte de los encuestados. En general, el cuestionario

6 Consideramos 37 comunas: Cerrillos, Cerro Navia, Conchalí, El Bosque, Estación Central, Huechuraba, Independencia, La Florida, La Granja, La Pintana, La Reina, Las Condes, Lo Barnechea, Lo Espejo, Lo Prado, Macul, Maipú, Nuñoa, Pedro Aguirre Cerda, Peñalolén, Providencia, Pudahuel, Puente Alto, Quilicura, Quinta Normal, Recoleta, Renca, San Bernardo, San Joaquín, San Miguel, San Ramón, Santiago y Vitacura. Excluimos solamente Padre Hurtado, Pirque y San José de Maipo por pertenecer más bien a áreas semirrurales y no tener relaciones muy estrechas con el resto de la capital.

7 En esta correlación nuevamente seguimos los datos de ICCOM (www.iccom.cl). 
hace énfasis en la obtención de explicaciones o justificaciones, con el fin de permitir un análisis cualitativo lo más refinado posible.

Las preguntas específicas que permitieron obtener los datos presentados en este artículo son las número 7 y 10, relativas a la similitud (7: “¿Qué lugares del país siente que hablan igual a usted?”; 10: “QQué lugares del país considera que hablan diferente a usted?"), las número 8 y 11 , relativas al agrado (8: “¿En qué lugar o lugares del país le gusta cómo se habla el español/castellano?”; 11: “¿En qué lugar o lugares del país no le gusta cómo se habla el español/castellano?"), y las número 9 y 12 , relativas a la calidad (9: “¿En qué lugar del país considera usted que hablan mejor?”; 12: “¿En qué lugar del país considera usted que hablan peor?"). Todas estas preguntas tienen respuestas de carácter abierto y, en el caso de las relativas al agrado y la calidad, permiten que los encuestados expliquen la razón de su respuesta.

El cuestionario fue aplicado a los sujetos por tres colaboradoras, licenciadas con especialización en Lingüística o profesoras de Lenguaje, entre marzo y noviembre del 2010. Las colaboradoras encuestaron a los individuos en sus lugares de trabajo, en sus hogares o en lugares públicos, poniendo por escrito las respuestas en el formulario, con total fidelidad a las palabras de cada encuestado. La aplicación de cada encuesta demoró 20 minutos en promedio.

\subsection{ANÁLISIS DE LOS DATOS}

Una vez finalizada la recolección de datos, estos fueron ingresados a un formulario electrónico (SurveyXact) desarrollado especialmente para el proyecto. Esta aplicación permite cruzar automáticamente las respuestas con las variables sociodemográficas.

La sección pertinente del cuestionario contenía únicamente preguntas de respuesta abierta, por lo que efectuamos primero un análisis cualitativo para generar categorías cuantificables y a continuación realizamos un análisis cuantitativo. En la constitución de categorías cuantificables, agrupamos las respuestas de tipo geográfico de acuerdo con las zonas políticoadministrativas mayores de vigencia oficial en Chile desde 1974: zona norte (regiones I a IV y la XIV, añadida en el 2007), zona central (regiones V a VII y Metropolitana), zona sur (regiones VIII y IX) y zona sur-austral (regiones X a XII y la XV, añadida en el 2007), las que además coinciden con sendas zonas geolingüísticas (Wagner 2006a). Cuando los encuestados mencionaron ciudades, las adscribimos a cada una de estas zonas; no obstante, en la presentación y análisis de resultados mencionamos qué ciudades aparecen individualizadas con frecuencia para cada una de ellas. Las respuestas de 
carácter distinto al geográfico, tales como las que indicaban zonas urbanas o que negaban similitudes, fueron adscritas a categorías independientes. Lo mismo hicimos con respuestas de tipo geográfico pero difícilmente adscribibles en términos estereotípicos a una zona, como sucede con Isla de Pascua (comunidad bilingüe y geográfica y culturalmente bastante distanciada del Chile continental, aunque oficialmente pertenece a la $\mathrm{V}$ Región, es decir, a la zona central).

\section{PRESENTACIÓN Y DISCUSIÓN DE RESULTADOS}

\subsection{SimiLitud}

\subsubsection{Lugares de Chile percibidos como de habla similar a la propia}

En la Tabla 2 se muestra dónde consideran los encuestados que se habla de una forma igual o parecida a como ellos lo hacen:

Tabla 2. Similitud: lugares de Chile donde se habla de manera similar

\begin{tabular}{|c|c|c|}
\hline & respondientes & porcentaje \\
\hline Zona central & 301 & $75,2 \%$ \\
\hline Zona sur & 28 & $7,0 \%$ \\
\hline Todo Chile & 23 & $5,7 \%$ \\
\hline Zona norte & 19 & $4,7 \%$ \\
\hline Ninguna parte & 12 & $3,0 \%$ \\
\hline No sabe/no responde & 17 & $4,2 \%$ \\
\hline total & 400 & $100 \%$ \\
\hline \multicolumn{2}{|l|}{}
\end{tabular}

La gran mayoría de las respuestas, como era esperable, se concentran en la zona central. Entre estas respuestas, un porcentaje muy importante corresponde a Santiago $(43,2 \%)$. Para la zona central se menciona con frecuencia, además, la V Región, con un protagonismo marcado de la ciudad de Valparaíso (4,2\%). En cuanto a la zona sur, la ciudad que se menciona con mayor frecuencia es Concepción (4\%), y para la zona norte, La Serena $(1,3 \%)$. Cabe destacar, por otra parte, que la opinión de que en todo Chile se habla igual a como se hace en la capital tiene una frecuencia no mucho 
menor que la que escoge la zona sur y levemente mayor que la que prefiere la zona norte.

\subsubsection{Lugares de Chile percibidos como de habla diferente a la propia}

En la Tabla 3 se muestra dónde consideran los encuestados que se habla de una forma distinta a como ellos lo hacen:

Tabla 3. Similitud: lugares de Chile donde se habla de manera diferente

\begin{tabular}{|l|c|c|}
\hline & respondientes & porcentaje \\
\hline Zona sur & 200 & $50,0 \%$ \\
\hline Zona norte & 100 & $25,0 \%$ \\
\hline Ninguna & 32 & $8,1 \%$ \\
\hline Zona central & 16 & $4,0 \%$ \\
\hline Todas partes & 15 & $3,4 \%$ \\
\hline $\begin{array}{l}\text { Lugares donde viven personas de } \\
\text { clase baja/alta en Santiago }\end{array}$ & 15 & $3,4 \%$ \\
\hline Enclaves rurales & 11 & $2,8 \%$ \\
\hline Isla de Pascua & 6 & $1,5 \%$ \\
\hline No sabe/no responde & 5 & 1,3 \\
\hline total & 400 & $100 \%$ \\
\hline
\end{tabular}

La zona sur es donde la mayor parte de los informantes cree que se habla distinto a la manera en que ellos lo hacen. Los lugares concretos que muestran frecuencia considerable son la isla de Chiloé $(9 \%)$ y la ciudad de Temuco $(3,8 \%)$. La zona norte tiene la segunda mayoría, con protagonismo especial de las ciudades de Arica (3,3\%) y Antofagasta (1\%). Tanto para la zona norte como para la zona sur hay un grupo de informantes que especifican que se refieren especialmente a los extremos respectivos del país. Las razones que se usan para escoger estas zonas, y las demás en general, aluden a que en ellas se habla "cantadito".

Cabe destacar que en este ítem, a diferencia de en el relativo a la percepción de igualdad, se inmiscuyen parámetros que no son en sentido estricto geográficos, sino sociodemográficos, tales como los recursos económicos y el grado de urbanización. 


\subsection{CALIDAD}

\subsubsection{Lugares de Chile considerados de mejor habla}

La Tabla 4 muestra en qué zonas del país los encuestados opinan que se habla de mejor forma:

Tabla 4. Calidad: lugares de Chile donde se habla mejor

\begin{tabular}{|l|c|c|}
\hline & respondientes & porcentaje \\
\hline Zona central & 224 & $56,0 \%$ \\
\hline Zona sur & 60 & $15,0 \%$ \\
\hline Zona norte & 58 & $14,5 \%$ \\
\hline Ninguna & 34 & $8,5 \%$ \\
\hline Todas partes & 6 & $1,5 \%$ \\
\hline Lugares donde viven personas de estrato alto & 5 & $1,3 \%$ \\
\hline Enclaves urbanos & 3 & $0,8 \%$ \\
\hline Enclaves costeros & 3 & $0,8 \%$ \\
\hline No sabe/no responde & 7 & $1,8 \%$ \\
\hline total & 400 & $100,0 \%$ \\
\hline
\end{tabular}

La gran mayoría opina que en la zona central se habla mejor que en el resto del país. La localidad que tiene mayor proporción de menciones es Santiago, con un $44 \%$ del total de respuestas. Las zonas sur y norte tienen porcentajes muy similares de respuestas, con un muy leve predominio de la primera de estas zonas. En la zona sur destacan las localidades de Concepción (1,5\%), la isla de Chiloé $(1,3 \%)$, Temuco (1\%) y Valdivia (1\%). En la zona norte, mientras tanto, sobresalen Arica $(3,8 \%)$ y La Serena (1\%). Un número considerable de informantes, finalmente, opina que en ninguna parte del país se habla mejor, sea porque consideran que "todos hablan mal" o bien porque "nadie habla peor o mejor, sino diferente".

Las razones aludidas para preferir Santiago (es decir, la gran mayoría de las de la zona central) son de dos tipos. Hay razones lingüísticas, tales como su neutralidad ("no tiene acento"), su vocabulario ("hay menos modismos", "la gente ocupa mejor las palabras") y su pronunciación ("hay mejor dicción", "modulamos más"). Por otra parte, la gran mayoría interpreta la pregunta “"por qué?”" como una indagación en los motivos (extralingüísticos) de que se hable mejor en la capital; la mayoría atribuye esta condición al mayor nivel educativo que perciben en este lugar, así como a su situación urbana y su carácter central desde el punto de vista administrativo. 
Los que atribuyen mejor hablar a la zona sur lo hacen principalmente por su fonética ("dicen íntegras las consonantes", "modulan mejor", "hablan más lento/pausado", "hablan más cantadito") y por su léxico ("las palabras son más castizas", "tiene menos modismos", "usan menos groserías", "se refieren a las cosas por su nombre", "no hay tanto extranjerismo").

Finalmente, y de manera muy similar a la zona sur, los informantes que opinan que se habla mejor en la zona norte atribuyen al habla de este lugar características como una mejor fonética ("pronuncian todas las letras", "modulan bien", "hablan más lento/pausado") y un mejor léxico ("vocabulario más rico", "usan pocos modismos", "usan las palabras mejor", "sin tantas groserías"). Sin embargo, a diferencia de la zona sur, varios informantes atribuyen el mejor hablar de la zona norte al contacto con las variedades peruana y boliviana.

\subsubsection{Lugares de Chile considerados de peor habla}

La Tabla 5 muestra en qué zonas del país los encuestados opinan que se habla de peor forma:

Tabla 5. Calidad: lugares de Chile donde se habla peor

\begin{tabular}{|l|c|c|}
\hline & respondientes & porcentaje \\
\hline Zona central & 140 & $35,0 \%$ \\
\hline Lugares donde viven personas de estrato bajo & 93 & $23,3 \%$ \\
\hline Zona sur & 71 & $17,8 \%$ \\
\hline Zona norte & 41 & $10,3 \%$ \\
\hline Ninguna & 17 & $4,3 \%$ \\
\hline Enclaves rurales & 12 & $3,0 \%$ \\
\hline Todas & 4 & $1,0 \%$ \\
\hline Enclaves urbanos & 2 & $0,5 \%$ \\
\hline Enclaves costeros & 1 & $0,3 \%$ \\
\hline Zona sur-austral & 1 & $0,3 \%$ \\
\hline No sabe/no responde & 18 & $4,5 \%$ \\
\hline total & 400 & $100,0 \%$ \\
\hline
\end{tabular}

La mayoría de los encuestados cree que se habla peor en la zona central, en particular en Santiago (18,3\%). No obstante, parte de las respuestas que aluden a la zona central mencionan comunas de la capital en que predominan los habitantes de estrato bajo: La Pintana (3\%), Cerro Navia, Pudahuel, Puente Alto, San Miguel (0,5\% cada una), Lo Espejo y San Ramón (0,3\% 
cada una). Igualmente, aunque no se mencionen comunas, muchas respuestas culpan del mal hablar de Santiago a los "flaites", esto es, a los individuos que encarnan el estereotipo del joven de estrato bajo normalmente vinculado al mundo delictual. Esto es congruente con lo hallado en el ítem relativo al desagrado de los informantes por el habla de zonas de Chile (cf. 4.3.2), en el sentido de que ambos casos revelan una proyección de la estratificación social sobre la geografía. Asimismo, apunta en la misma dirección el hecho de que la segunda mayoría de respuestas en este ítem corresponda a las "poblaciones" (barriadas populares) o zonas "periféricas" y "marginales" de los núcleos urbanos del país, en lugar de a una zona o localidad particular.

La gran mayoría de quienes opinan que en la zona central y en especial en Santiago se habla peor ejemplifican el mal hablar con rasgos de tipo léxico: principalmente, excesivo uso de groserías y modismos e influencia del coa (jerga delictual chilena). Además, se mencionan factores fónicos como la pronunciación ("no se modula", "no pronunciamos las eses finales") y la excesiva velocidad para hablar.

Los encuestados que atribuyen peor hablar a los lugares donde habitan personas de estrato bajo, al igual que en el caso de la zona central, apuntan mayormente al vocabulario: abuso de expresiones groseras y modismos, influencia del coa y pobreza léxica. En segundo lugar, factores fónicos: falta de modulación, pérdida del momento oclusivo de $/ \mathrm{t} \mathrm{f} /$, "sonsonetes que no corresponden" y "pronunciación exagerada". Por último, algunos tratan de explicar los motivos de que se hable mal y aluden a la falta de educación, la pobreza, la delincuencia y la drogadicción.

\subsection{Agrado}

\subsubsection{Agrado por el habla de lugares de Chile}

La Tabla 6 muestra en qué zonas del país a los encuestados les gusta cómo se habla:

Tabla 6. Agrado: lugares de Chile donde se habla de manera agradable

\begin{tabular}{|l|c|c|}
\hline & respondientes & porcentaje \\
\hline Zona central & 187 & $46,8 \%$ \\
\hline Zona sur & 128 & $32,0 \%$ \\
\hline Zona norte & 46 & $11,5 \%$ \\
\hline Ninguno & 15 & $3,8 \%$ \\
\hline
\end{tabular}




\begin{tabular}{|l|c|c|}
\hline Todas partes & 10 & $2,5 \%$ \\
\hline Enclaves rurales & 3 & $0,8 \%$ \\
\hline Isla de Pascua & 2 & $0,5 \%$ \\
\hline No sabe/no responde & 9 & 2,3 \\
\hline total & 400 & $100,0 \%$ \\
\hline
\end{tabular}

Las localidades de la zona central son aquellas cuya forma de hablar agrada mayoritariamente a los informantes de la capital. La ciudad que más menciones tiene es Santiago, con un 31,5\% del total de las respuestas. La segunda mayoría la tiene la zona sur. Destacan localidades específicas como la isla de Chiloé (4,3\%), Temuco (1,8\%), Valdivia (1,8\%), Concepción $(1,3 \%)$ y Punta Arenas (1\%). En la zona norte, finalmente, sobresalen Arica (2\%) y La Serena $(1,3 \%)$.

Las razones por las que se prefieren estos lugares son diversas. El agrado por Santiago, en primer lugar, se debe a que se percibe en su habla, en proporciones idénticas, neutralidad de acento ("no es tan cantadito") y corrección idiomática (especialmente en comunas de estrato socioeconómico alto), así como, en menor grado, claridad ("es más entendible"). También algunos informantes señalan identificarse más con esta forma de hablar por considerar que es más "puro", es decir, menos mezclado con las variedades peruana o boliviana, por ejemplo.

La forma de hablar de la zona sur es agradable para los informantes fundamentalmente por su entonación ("es cantadito/musical"). Además se percibe y evalúa favorablemente que sea un habla marcadamente afectiva ("es cariñosa" "es más acogedora", "es más amoroso el idioma"). En menor grado a los encuestados les gusta el habla de la zona sur por su léxico (en particular, amplitud de repertorio y precisión: "todo tiene su nombre", "ocupan más vocabulario que nosotros", etc.) y por su corrección idiomática. En el caso particular de Chiloé, de manera muy similar, se menciona principalmente su entonación ("es cantadito/musical"), su léxico diferencial (arcaísmos, por ejemplo) y su afectividad.

Entre los sujetos que prefieren la zona norte, por último, el argumento predominante es que se trataría de una forma de hablar más correcta que las demás. Varios encuestados atribuyen esta corrección a la influencia de las variedades altiplánicas vecinas (español peruano y boliviano).

\subsubsection{Desagrado por el habla de lugares de Chile}

La Tabla 7 muestra en qué zonas del país a los encuestados no les gusta como se habla: 
Tabla 7. Agrado: lugares de Chile donde se habla de manera desagradable

\begin{tabular}{|l|c|c|}
\hline & respondientes & porcentaje \\
\hline Zona central & 128 & $32,0 \%$ \\
\hline Zona sur & 76 & $19,0 \%$ \\
\hline Lugares donde viven personas de estrato bajo/alto & 63 & $15,8 \%$ \\
\hline Zona norte & 56 & $14,0 \%$ \\
\hline Ninguno & 45 & $11,3 \%$ \\
\hline Enclaves rurales & 9 & $2,3 \%$ \\
\hline Todas partes & 2 & $0,5 \%$ \\
\hline Zona sur-austral & 2 & $0,5 \%$ \\
\hline Enclaves urbanos & 1 & $0,3 \%$ \\
\hline Enclaves costeros & 1 & $0,3 \%$ \\
\hline No sabe/no responde & 17 & 2,3 \\
\hline total & 400 & $100,0 \%$ \\
\hline
\end{tabular}

A la mayoría de los encuestados les disgusta cómo se habla en la zona central, principalmente en Santiago (16,3\%). Esto puede parecer contradictorio con las actitudes positivas predominantes hacia estos mismos lugares. Sin embargo, debe tenerse en cuenta que el desagrado de los informantes parece deberse más bien a la proyección de la estratificación social sobre la geografía de la capital. Con frecuencia hacen la especificación de que les desagrada la forma de hablar de los sectores populares de la capital, especialmente los enclaves considerados propios del estrato bajo (como la comuna de La Pintana o "poblaciones", es decir, barriadas populares, tales como La Legua). A esto debe sumarse la consideración de que una proporción importante de las respuestas no alude a ninguna localidad en particular, sino a "zonas marginales" y divisiones geográficas de base social similares. Por supuesto, a quienes les desagrada la forma de hablar de los sectores populares son mayoritariamente de estrato medio y alto; a la inversa, quienes miran con desprecio los sectores acomodados ("porque no expresan la identidad chilena") son de estrato bajo.

La zona sur se ubica en segunda posición. En particular, se mencionan con frecuencia localidades como Temuco $(1,8 \%)$ y la isla de Chiloé $(1,8 \%)$. A continuación, muy de cerca, siguen los lugares asociados a los estratos populares (también hay una mención a los vinculados al estrato alto) y la zona norte. De esta última zona destaca Arica (3,5\%).

El desagrado por la forma de hablar de la zona central, y de Santiago en particular, se debe principalmente a su vocabulario. Se considera que este se encuentra plagado de modismos y groserías, así como especialmente 
afectado por el coa, jerga delictual chilena que se asocia con frecuencia a los estratos bajos. Además, se hace mención de su pronunciación, aludiendo a falta de modulación, excesiva velocidad para hablar y rasgos concretos como el debilitamiento de /s/ implosiva y la pérdida del momento oclusivo de $/ \mathrm{t} \mathrm{f}$, rasgo, este último, estigmatizado y asociado fuertemente al estrato bajo. Por otra parte, uno de los informantes que valora negativamente el habla de los sectores acomodados señala el hablar "con una papa en la boca", es decir, con modulación muy relajada y cierto grado de posteriorización vocálica. Esta forma de hablar constituye un estereotipo que se usa para caracterizar el habla de algunos individuos del estrato alto. Finalmente, a algunos encuestados les desagrada el habla de la zona central por el poco respeto o educación que se usa para comunicarse.

El habla de la zona sur se considera desagradable fundamentalmente por razones fónicas, tales como su entonación "muy cantadita" y su pronunciación ("se comen letras", "no modulan bien"). En segundo lugar se mencionan hechos extralingüísticos, como que su habla es muy "ahuasada", es decir, tiene rasgos propios del habla campesina, y que está afectada por la mezcla con el mapudungún (lengua de los mapuches), especialmente en Temuco.

El desagrado por el habla de la zona norte se debe principalmente a que se percibe como afectada por el contacto con las variedades vecinas del español andino (Perú y Bolivia), por lo cual "hablan como peruanos/ bolivianos". En menor grado, se menciona su léxico ("tienen muchos modismos", "tienen otros modismos, que no se entienden", "tienen poco vocabulario"), su pronunciación ("difícil de entender", "no modulan", "se comen las palabras") y su entonación "cantadita, como la de los peruanos".

Por último, las razones para evaluar negativamente el habla de sectores populares (no necesariamente de la capital, sino que en general de zonas urbanas) son predominantemente léxicas: excesivo uso de groserías e influjo del coa (jerga delictual chilena). Además se menciona su pronunciación, en particular la falta de modulación.

\subsection{DifERENCIAS DE ACUERDO CON VARIABLES EXTRALINGÜÍSTICAS}

De las variables extralingüísticas relativas al perfil sociodemográfico de los encuestados, solamente el estrato socioeconómico mostró influencia en las respuestas de carácter positivo. En la Tabla 8 se presentan los resultados de las valoraciones positivas para cada una de las dimensiones consideradas en nuestro estudio, separadas por cada uno de los estratos (bajo, medio y alto): 
Tabla 8. Valoraciones positivas de acuerdo con estrato socioeconómico

\begin{tabular}{|c|c|c|c|c|c|c|c|}
\hline & \multicolumn{2}{|c|}{ Bajo } & \multicolumn{2}{|c|}{ Medio } & \multicolumn{2}{|c|}{ Alto } \\
\hline & & resp. & $\%$ & resp. & $\%$ & resp. & $\%$ \\
\hline \multirow{8}{*}{ 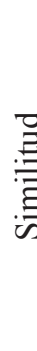 } & Zona norte & 10 & 5,6 & 9 & 5 & 0 & 0 \\
\hline & Zona central & 107 & 59,4 & 154 & 85,6 & 40 & 100 \\
\hline & Zona sur & 24 & 13,3 & 9 & 5 & 0 & 0 \\
\hline & Todos & 22 & 12,2 & 2 & 1,1 & 0 & 0 \\
\hline & Ninguno & 10 & 5,6 & 2 & 1,1 & 0 & 0 \\
\hline & Enclaves urbanos & 0 & 0 & 4 & 2,2 & 0 & 0 \\
\hline & Otros & 7 & 3,9 & 0 & 0 & 0 & 0 \\
\hline & Total & 180 & 100 & 180 & 100 & 40 & 100 \\
\hline \multirow{8}{*}{ 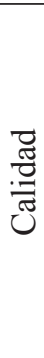 } & Zona norte & 18 & 10 & 40 & 22,2 & 1 & 2,5 \\
\hline & Zona central & 98 & 54,4 & 99 & 55 & 33 & 82,5 \\
\hline & Zona sur & 30 & 16,7 & 26 & 14,4 & 3 & 7,5 \\
\hline & Todos & 2 & 1,1 & 3 & 1,7 & 0 & 0 \\
\hline & Ninguno & 23 & 12,8 & 7 & 3,9 & 2 & 1,1 \\
\hline & Enclaves urbanos & 0 & 0 & 3 & 1,7 & 0 & 0 \\
\hline & Otros & 9 & 5 & 2 & 1,1 & 1 & 6,4 \\
\hline & Total & 180 & 100 & 180 & 100 & 40 & 100 \\
\hline \multirow{8}{*}{ 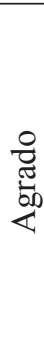 } & Zona norte & 10 & 5,6 & 31 & 17,2 & 3 & 7,5 \\
\hline & Zona central & 88 & 48,9 & 71 & 39,4 & 26 & 65 \\
\hline & Zona sur & 51 & 28,3 & 66 & 36,7 & 11 & 27,5 \\
\hline & Todos & 6 & 3,3 & 4 & 2,2 & 0 & 0 \\
\hline & Ninguno & 8 & 4,4 & 6 & 3,3 & 0 & 0 \\
\hline & Enclaves rurales & 3 & 1,7 & 0 & 0 & 0 & 0 \\
\hline & Otros & 14 & 7,8 & 2 & 1,2 & 0 & 0 \\
\hline & Total & 180 & 100 & 180 & 100 & 40 & 100 \\
\hline
\end{tabular}

En primer lugar, se puede apreciar que, en la dimensión de la similitud, a medida que se asciende en la escala socioeconómica, la actitud positiva hacia el habla de la zona central es más generalizada: los individuos de estrato alto nunca hacen valoraciones de otras zonas en este ítem. Cabe destacar, en relación con esta misma dimensión, que un 35\% de los sujetos de este estrato mencionan comunas de la ciudad de Santiago que son consideradas aquellas en que habitan las personas de clase alta: Vitacura, Las Condes, Providencia, o bien hacen alusión al sector oriente o alto (hacia la cordillera de los Andes) de la capital ("de Plaza Italia hacia arriba"), donde se encuentran concentradas estas comunas. Esta especificación la hacen igualmente en las dimensiones de la calidad (25\%) y del agrado (20\%). Por 
el lado contrario, la valoración positiva de la zona sur en la dimensión de la similitud incrementa su frecuencia a medida que se desciende en la escala socioeconómica, aunque siempre sigue siendo mayoritaria la preferencia por el centro. Tendencia similar muestran las opciones que niegan o absolutizan las diferencias, más frecuentes a medida que se desciende en la escala social.

En segundo lugar, en la dimensión de la calidad la zona norte concentra la mayoría de sus respuestas positivas en el estrato socioeconómico medio, mientras que la opción que niega que en algún lugar de Chile se hable bien aumenta su frecuencia a medida que se desciende en la escala social.

En tercer lugar, en la dimensión del agrado, nuevamente la zona norte tiene su mayor preferencia entre sujetos de estrato medio.

\subsection{Discusión}

Los datos obtenidos respecto de la percepción de similitud (o diferencia) lingüística entre el habla de los encuestados y la de otros lugares de Chile revelan de manera clara, y de acuerdo con lo esperable, que la zona central, especialmente Santiago y la Región Metropolitana en general, es considerado el lugar donde se habla más parecido a cómo lo hacen los encuestados; en cambio, la zona sur y la zona norte, en especial la primera de estas zonas (y en particular enclaves como Chiloé), son consideradas distintas lingüísticamente a Santiago. Interpretamos estos datos como signo de que, dentro del panorama diatópico chileno, los santiaguinos sienten una fuerte identificación con la propia forma de hablar, $\mathrm{y}$, consecuentemente, poseen un alto grado de autoestima lingüística. La autoestima es especialmente acentuada entre los individuos de estrato alto, como muestran los datos presentados en la sección 4.4. En este sentido, las actitudes lingüísticas de los santiaguinos hacia el habla de la zona central, y de Santiago, con especial importancia, son de carácter positivo, en contraste con la actitud tendiente hacia el polo negativo revelada por el distanciamiento lingüístico que perciben en las demás zonas del país.

Igualmente en cuanto a la dimensión de la similitud, llama la atención la relativamente importante frecuencia con que se minimizan las diferencias lingüísticas en el territorio chileno: hay una cantidad no despreciable de informantes que opinan que en todo Chile se habla igual, así como un número importante que piensa que en ninguna parte de Chile se habla muy distinto a la capital. Como se pudo ver en la Tabla 2, ambas opiniones totalizan un $8,7 \%$ de las respuestas, lo que posicionaría esta opinión en el segundo lugar general en jerarquía de frecuencia. En la Tabla 3, la opinión de que en ninguna parte de Chile se habla distinto a como se hace en Santiago ocupa un 
$8,1 \%$ de las respuestas, con lo cual se ubica en el tercer lugar de frecuencia. Sería interesante explorar la posibilidad de que esto sea manifestación de la operación semiótica denominada elisión (erasure) por Irvine y Gal (2000), operación normalmente llevada a cabo por los grupos que ostentan una posición hegemónica o central en una comunidad:

Erasure is the process in which ideology, in simplifying the sociolinguistic field, renders some persons or activities (or sociolinguistic phenomena) invisible. Facts that are inconsistent with the ideological scheme either go unnoticed or get explained away. So, for example, a social group or a language may be imagined as homogeneous, its internal variation disregarded. (Irvine y Gal 2000: 38)

Por otra parte, podría pensarse que esta apreciación tiene fundamento en la relativamente poca saliencia perceptual de la diferenciación geolingüística chilena (Moreno Fernández 2009: 274), lo cual se condice con la debilidad con que las isoglosas pertinentes permiten diferenciar a los especialistas entre regiones lingüísticas de Chile (Wagner 2006a: 19).

Las dimensiones de la calidad y el agrado muestran patrones de opinión que podrían considerarse internamente contradictorios: el lugar donde se piensa que se habla "mejor" y de manera más agradable para los encuestados es la zona central, en particular Santiago, pero al mismo tiempo es donde se cree que se habla "peor" y de modo más desagradable. Para explicar esta aparente contradicción debe tomarse en cuenta que cuando una parte importante de los encuestados abundan en explicaciones sobre qué lugar específico de la zona central les parece de habla peor o más desagradable aluden a comunas estereotípicas del estrato sociocultural bajo, o bien aclaran que se trata de una opinión no sobre todos los hablantes de la zona central, sino sobre los individuos de estrato sociocultural bajo que habitan en esta zona, sobre todo el personaje urbano estereotipado denominado flaite $^{8}$. Entonces, cuando la mayor parte de los santiaguinos encuestados muestran actitudes positivas, mediante la atribución de "buen" hablar y manifestación de agrado, hacia el habla de su propia comunidad, se presentan a sí mismos como un grupo homogéneo y de conducta digna de aprecio. En cambio, cuando se trata de atribuir cualidades negativas, particularizan y estigmatizan a un "otro" que forma parte de su hábitat lingüístico pero con el cual no se sienten identificados (aunque el individuo respondiente sea de estrato

\footnotetext{
8 'Persona de clase social baja y comportamiento y apariencia extravagante, que es relacionada generalmente con el mundo delictual' (Academia Chilena de la Lengua 2010: s. v.).
} 
sociocultural bajo). En este sentido, los santiaguinos, en su percepción y valoración de la variación geográfica de Chile, proyectan la identificación de diferencias socioculturales hacia el plano geográfico: las diferencias geolingüísticas del español de Chile, especialmente las internas a la urbe donde viven, son racionalizadas en términos de la diferente procedencia sociocultural de las personas que ocupan dichos espacios. Coincide con este patrón de pensamiento la no poco frecuente identificación del habla rural, de ninguna zona en particular, como una de aquellas consideradas de peor hablar o desagradable. Concuerda, asimismo, con la especificación que hacen a menudo los sujetos de estrato alto respecto de sus valoraciones positivas, en el sentido de que las restringen a las comunas de la capital donde se sabe que habitan personas de clase alta. Todo esto es coherente con lo que señala Moreno Fernández (2009) respecto de la superposición de la variación lingüística sociocultural en la variación geográfica chilena, lo que en su opinión se explica por las particularidades de la historia social del español en Chile:

En el siglo XIX, tras la Independencia, se fue fraguando un pensamiento lingüístico basado en la codificación propuesta por Andrés Bello y difundido a través de una legislación, unas ordenanzas educativas y unas instituciones que oficializaban la lengua española o castellana como vehículo de enseñanza. La escolarización sistemática de la población hizo posible la difusión de un modelo de lengua y todo ello tuvo que influir en el nacimiento de una conciencia y de unas actitudes gobernadas más por parámetros sociolingüísticos que geolingüísticos (Moreno Fernández 2009: 271).

El sur de Chile muestra un incremento notable de valoraciones positivas en la dirección similitud $\rightarrow$ calidad $\rightarrow$ agrado, es decir, en dirección hacia la dimensión afectiva. En las tres dimensiones ocupa el segundo lugar en jerarquía, después de la zona central; sin embargo, la diferencia entre ellas es de 68,2 puntos porcentuales en similitud, de 41 en calidad y de solo 14,8 en agrado. Puede concluirse entonces, que la actitud de los santiaguinos hacia el habla de la zona sur mejora en la medida en que se encuentren involucrados factores de tipo afectivo, mientras que disminuye en el grado en que aparezcan elementos de orden cognitivo.

Finalmente, en cuanto a las razones que dan los encuestados para justificar sus valoraciones, debemos destacar que, tanto en la dimensión de la calidad como en la dimensión del agrado, estas razones tienen estrecha relación con el concepto de corrección idiomática. En el marco de esta misma investigación, pero a partir de otras preguntas del cuestionario utilizado, 
hemos logrado esbozar un perfil del "español correcto" ideal que funciona entre los santiaguinos como modelo idiomático:

En síntesis, la idea del hablar correctamente el español reflejada transversalmente en las opiniones de los sujetos encuestados en Santiago de Chile consiste en pronunciar "bien" (es decir, con fonética de tierras altas, con realización plena de consonantes, más ajustada a la escritura), hablar con un ritmo pausado, con volumen considerable de la voz y sin un acento marcado. El vocabulario debe ser amplio, aunque debe ajustarse al recogido en el Diccionario académico y debe excluir las voces marcadas diasistemáticamente (coloquialismos, regionalismos, voces jergales, etc.), así como las groserías y las muletillas. El vocabulario, además, debe usarse con precisión desde el punto de vista del significado. Debe existir un respeto por reglas gramaticales, excluyendo las construcciones o variantes morfológicas consideradas subestándar o coloquiales. Por otro lado, el buen hablante debe tener en cuenta las normas académicas (de la RAE), así como expresarse de manera clara y adecuada a contexto (Rojas, en preparación).

Las razones que dan los sujetos para considerar "mejor" o "agradable" la forma de hablar de un lugar de Chile coinciden con este perfil lingüístico: consonantismo conservador, baja frecuencia de modismos y groserías, riqueza de vocabulario, neutralidad de acento, modulación cuidada y ritmo pausado, entre otras. Más aún, en el caso de la dimensión del agrado aparece de manera explícita la atribución de corrección idiomática y de claridad (dos cualidades muy relacionadas en la mente de los encuestados, según hemos podido comprobar; Rojas, en preparación) como fundamento de la valoración, cuando lo esperable era que estas características tuvieran presencia más bien en la dimensión de la calidad.

La única excepción a esta tendencia ocurre al justificar la valoración positiva del habla de la zona sur de Chile: el hablar "cantadito" contradice la neutralidad de acento del modelo idiomático de corrección, a pesar de lo cual los sujetos usan esta característica como argumento para considerar no solamente más agradable el habla de esta zona, sino también de mejor calidad. Paradójicamente, al justificar el agrado por el habla de Santiago se usa el argumento de valor contrario: su supuesta neutralidad de acento. Asimismo, cuando se justifica el desagrado por el habla del mismo sur también algunos sujetos usan el argumento de que se habla "cantadito". En general, en la justificación del agrado por la zona sur ocupa un lugar predominante la dimensión afectiva de las actitudes (compárese con la mayor prominencia de valoraciones positivas de la zona sur en la dimensión del agrado, según vimos antes), lo cual explicaría esta "suspensión" de la 
influencia del modelo idiomático de corrección, que corresponde más bien a la faceta cognitiva.

En cuanto a las razones que se emplean para fundamentar una valoración negativa en la dimensión del agrado, aparecen dos tópicos que no se encuentran en el resto de las argumentaciones: el carácter rural ("ahuasado") percibido en el habla de la zona sur y el carácter mixto de las hablas de la zona norte y de la zona sur; en el caso de la zona norte, se trata de una visión negativa de la mezcla con lo extranjero (hablas peruana y boliviana), mientras que en el caso de la zona sur el objeto de visión negativa es el contacto entre el español y el mapudungún. Ambos juicios revelan que el agrado sentido por una forma de hablar se encuentra asociado a la pureza que se percibe en él. Se trata de manifestaciones de la ideología del purismo lingüístico, de acuerdo con la cual los sujetos de una comunidad lingüística consideran deseable mantener la lengua libre de elementos extranjeros o indeseables (Langer y Davies 2005).

\section{CONCLUSIONES}

Los santiaguinos encuestados muestran actitud lingüística predominantemente positiva hacia el español de su propia zona, con la salvedad de los enclaves de estrato social bajo y rurales de este lugar, que son objeto de una apreciación negativa. La actitud positiva se manifiesta en las tres dimensiones valorativas del habla consideradas en nuestra investigación: similitud, calidad y agrado, aunque con especial nitidez en la primera de ellas, y se acentúa a medida que se asciende en la escala socioeconómica. En este sentido, los resultados son coherentes con el gran potencial de influencia político-cultural (Wagner 2006b; $c f$. también Oroz 1966: 49 y Prieto 1995-1996: 380-382) que tiene Santiago dentro de Chile, por ser el principal centro político-administrativo, económico, cultural y demográfico del país. Los habitantes de este lugar, de manera consecuente, muestran una gran autoestima lingüística cuando comparan su propia forma de hablar con las de los demás lugares del territorio nacional. La única tendencia que contrapesa la preeminencia valorativa de la zona central es el incremento de la valoración positiva de la zona sur especialmente en el dominio afectivo (agrado) y entre los segmentos medios y bajos de la escala socioeconómica. Sin embargo, este incremento nunca le permite alcanzar ni superar a la zona central del país en la estimación positiva de los sujetos encuestados. 
Entre las interrogantes que se pueden plantear a partir de nuestros hallazgos, consideramos de especial importancia las siguientes:

1. La actitud positiva del habla de Santiago y la zona central, ¿se da también entre los habitantes de otras zonas de Chile?

2. De ser afirmativa la respuesta a lo anterior, ¿han jugado o juegan algún papel los medios de comunicación de masas en la percepción del habla santiaguina por parte del resto del país?

3. ¿Existe un estereotipo lingüístico del habla santiaguina, sea en la capital o en otros lugares de Chile?

Además, en nuestra investigación han asomado a la luz otros asuntos en los que resultaría de interés profundizar, tales como 1) la posible manifestación del proceso semiótico-ideológico de elisión en la minimización de las diferencias geolingüísticas internas al español de Chile por parte de los santiaguinos, 2) la influencia del grado de urbanización (oposición habla urbana / habla rural) y el nivel sociocultural que se atribuye a los habitantes de ciertas zonas chilenas al momento de valorar su forma de hablar, y 3) la asociación del habla de la zona sur de Chile ( $y$ y de otras regiones no centrales?) con valoraciones positivas en el dominio afectivo.

Por último, en nuestro estudio se pueden vislumbrar algunos elementos de la zonificación lingüística popular (folk) de los santiaguinos. En principio, la mayor parte de los sujetos encuestados parecen distinguir fundamentalmente tres zonas: norte, centro y sur, cada una de ellas con sus ciudades o regiones representativas o más salientes. Esto coincide parcialmente con lo hallado desde el punto de vista dialectológico por Wagner (2006a), quien distingue entre zona norte, zona central, zona sur y zona sur-austral. Con la ayuda de los métodos usados en los estudios de dialectología perceptual (Preston 1999) podrían obtenerse datos de mayor fuerza y comparables con los relativos a actitudes recogidos en la presente investigación.

\section{REFERENCIAS BIBLIOGRÁFICAS}

Academia Chilena de la Lengua. 2010. Diccionario de uso del español de Chile (DUECh). Santiago: MN Editorial.

Bayard, D., A. Weatherall, C. Gallois y J. Pittam. 2001. Pax Americana?: Accent attitudinal evaluations in New Zealand, Australia and America. Journal of Sociolinguistics 5: 22-49.

Blas Arroyo, José Luis. 1995. De nuevo el español y el catalán, juntos y en contraste. Estudio de actitudes lingüísticas. Sintagma 7: 29-41. 
1999. Las actitudes hacia la variación intradialectal en la sociolingüística hispánica. Estudios Filológicos 34: 47-72.

Coupland, Nikolas y Adam JaWorski. 2004. Sociolinguistic Perspectives on Metalanguage: Reflexivity, Evaluation and Ideology. En Nikolas Coupland, Adam Jaworski y D. Galasiński (eds.). Metalanguage: Social and Ideological Perspectives, pp. 15-51. Berlín: Mouton de Gruyter.

Coseriu, Eugenio. 1990. El español de América y la unidad del idioma. En Actas del I Simposio de Filología Iberoamericana, pp. 43-75. Zaragoza: Pórtico.

1993. Competencia lingüistica y criterios de corrección. Apuntes del curso intensivo de perfeccionamiento dictado por el prof. Dr. Eugenio Coseriu (19 a 22 de octubre de 1987). Edición de Alfredo Matus y José Luis Samaniego. Santiago: Pontificia Universidad Católica de Chile.

Del Valle, José y Luis Gabriel-Stheeman. 2002. Nationalism, hispanismo and monoglossic culture. En José del Valle y Luis Gabriel-Stheeman (eds.): The Battle over Spanish between 1800 and 2000. Language ideologies and Hispanic intellectuals, pp. 1-13. Londres/ Nueva York: Routledge.

EDWards, John. 2009. Language and Identity. Cambridge: Cambridge University Press. 2011. Challenges in the Social Life of Language. Londres: Palgrave Macmillan.

Figueroa, Mauricio. 2007. Prestigio de las variantes de [tr] en la comuna de Concepción. Estudio sociolingüístico. En Actas del XVII Congreso de Investigación y Enseñanza de la Lingüística [http://www2.udec.cl/sochil2007/figueroa.pdf, fecha de consulta: 15 de agosto de 2011].

Gallardo, Andrés. 2009-2010. Andrés Bello y la unidad del idioma. Boletín de la Academia Chilena de la Lengua 79: 175-183.

Garrett, Peter. 2001. Language Attitudes and Sociolinguistics. Journal of Sociolinguistics 5(4): 626-631.

2010. Attitudes to language. Cambridge: Cambridge University Press.

Garrett, Peter, Angie Williams y Betsy Evans. 2005. Attitudinal data from New Zealand, Australia, the USA and the UK about each other's Englishes: Recent changes or consequences of methodology? Multilingua 24: 211-235.

GonzÁlez Martínez, JuAn. 2008. Metodología para el estudio de las actitudes lingüísticas. En Inés Olza Moreno, Manuel Casado Velarde y Ramón González Ruiz (eds.). Actas del XXXVII Simposio Internacional de la Sociedad Española de Lingüística (SEL), pp. 229238. Pamplona: Servicio de Publicaciones de la Universidad de Navarra.

HAENSCH, GÜNTHER. 2000. Introducción. En Textos clásicos para la historia de la lexicografía del español en América [CD-ROM, Colección Clásicos Tavera]. Madrid: Fundación Histórica Tavera/ Fundación MAPFRE.

IrVine, Judith y Susan GaL. 2000. Language Ideology and Linguistic Differentiation. En Paul V. Kroskrity (ed.). Regimes of Language: Ideologies, Politics, and Identities, pp. 35-84. Oxford: Oxford University Press.

KRISTIANSEN. TORE. 2010. Conscious and subconscious attitudes towards English influence in the Nordic countries: evidence for two levels of language ideology. International Journal of the Sociology of Language 204: 59-95.

Long, Daniel y Dennis Preston (eds.). 2002. Handbook of perceptual dialectology. Vol. II. Amsterdam: John Benjamins.

Langer, Nils y Winifred Davies. 2005. An Introduction to linguistic purism. En Nils Langer y Winifred Davies (eds.). Linguistic purism in the Germanic languages, pp. 1-17. Berlín: Walter de Gruyter.

MenÉndez Pidal, Ramón. 1945. La unidad del idioma. En Castilla, la tradición y el idioma, pp. 172-218. Buenos Aires: Espasa-Calpe. 
Mirroy, James. 2001. Language ideologies and the consequences of standardization. Journal of Sociolinguistics 5(4): 530-555.

Milroy, James y Lesley Milroy. 1999. Authority in Language: Investigating Standard English. Londres: Routledge.

Moreno Fernández, Francisco. 2009. La lengua española en su geografía. Madrid: Arco/ Libros.

Myers, David. 2010. Social Psychology. 10. a ed. Nueva York: McGraw-Hill.

Oroz, Rodolfo. 1966. La lengua castellana en Chile. Santiago: Universidad de Chile.

Preston, Dennis (ed.). 1999. Handbook of perceptual dialectology. Vol. I. Amsterdam: John Benjamins.

2002. Language with an Attitude. En J. K. Chambers, Peter Trudgill y Natallie Schilling-Estes (eds.). The Handbook of Language Variation and Change, pp. 40-66. Malden: Blackwell.

Prieto, LuIs. 1995-1995. Análisis sociolingüístico del dequeísmo en el habla de Santiago de Chile. Boletín de Filología de la Universidad de Chile XXXV: 379-452.

RoJAS, DARío. En preparación. Actitudes lingüísticas de hispanohablantes de Santiago de Chile: creencias sobre la corrección idiomática.

Schieffelin, Bambi, Kathryn Woolard y Paul Kroskrity (eds.). 1998. Language Ideologies: Practice and Theory. Nueva York/Oxford: Oxford University Press.

TASSARA, Gilda. 1992. Actitudes lingüísticas ante la variación de /ch/. Revista de Lingüistica Teórica y Aplicada 30: 263-271.

1993-1994. Valoración subjetiva de usos fonéticos alternativos en una muestra porteña. Nueva Revista del Pacifico 38/39: 143-157.

Trudgill, Peter y J. M. Hernández. 2007. Diccionario de sociolingüística. Madrid: Gredos.

Valdivieso, Humberto. 1981. Valoración subjetiva de los usos lingüisticos, Concepción: Universidad de Concepción, Facultad de Humanidades y Arte.

1983. Prestigio y estigmatización: factor determinante en la enseñanza institucionalizada de la lengua materna. Revista de Lingüistica Teórica y Aplicada 21: 137-142.

Wagner, Claudio. 2006a. Las zonas dialectales de Chile. Revista de Lingüística Teórica y Aplicada 44: 13-29. 41: 277-284.

Woolard, Kathryn. 1998. Introduction. En Bambi Schieffelin, Kathryn Woolard y Paul Kroskrity (eds.). Language Ideologies: Practice and Theory, pp. 3-47. Nueva York: Oxford University Press.

Zahn, Christopher y Robert Hopper. 1985. Measuring language attitudes: the speech evaluation instrument. Journal of Language and Social Psychology 4: 113-123.

ZimMERMANn, Klaus. 2008. La invención de la norma y del estándar para limitar la variación lingüística y su cuestionamiento actual en términos de pluricentrismo (mundo hispánico). En Jürgen Erfurt y Gabriele Budach (eds.). Standardisation et déstandardisation. Le français et l'espagnol au XXe siècle / Estandarización y desestandarización. El francés y el español en el siglo XX, 187-208. Frankfurt am Main: Peter Lang. 